This item is the archived peer-reviewed author-version of:

Petri-net based cascading effect analysis of vapor cloud explosions

\title{
Reference:
}

Zhou Jianfeng, Reniers Genserik.- Petri-net based cascading effect analysis of vapor cloud explosions

Journal of loss prevention in the process industries - ISSN 0950-4230 - 48(2017), p. 118-125

Full text (Publisher's DOI): https://doi.org/10.1016/J.JLP.2017.04.017

To cite this reference: https://hdl.handle.net/10067/1427110151162165141 


\title{
A Petri-net based simulation analysis approach for cascading effect of vapor
}

\section{cloud explosions}

\author{
Jianfeng Zhou ${ }^{\text {a, *, Genserik Reniers }}{ }^{\text {b, c, d }}$
}

a. School of Electromechanical Engineering, Guangdong University of Technology, Guangzhou 510006, China

b. Faculty of Technology, Policy and Management, Safety and Security Science Group (S3G), TU Delft, 2628 BX Delft, The Netherlands

c. Faculty of Applied Economics, Antwerp Research Group on Safety and Security (ARGoSS), Universiteit Antwerpen, 2000 Antwerp, Belgium

d. CEDON, KULeuven, 1000 Brussels, Belgium

\begin{abstract}
If flammable gas is mixed with air and ignited, it is easy to form vapor cloud explosion (VCE) which is destructive, and easy to trigger the domino effect because of its large extent of impact. A VCE accident may induce secondary VCE accidents, then tertiary VCE accidents, and so on. This is called the cascading effect of VCE accidents, which is capable of causing severe damages to human, assets, and the environment, thus requiring an understanding of probabilities and propagation patterns thereof to prevent and mitigate the potential damages. In this work, a novel methodology for probability analysis of VCE cascading effect based on Petri-net is proposed. According to the limits of states and their changes which reflect characteristics of VCE propagation, the improvement of Petri-net is provided. The modeling approach and analysis process of VCE cascading effect are discussed. The application and efficacy of the methodology are demonstrated via an example of VCE accidents occurring in a gasoline tanks area. The results show that the developed methodology can effectively reveal the propagation patterns of VCE cascading and calculate the respective probabilities of VCE accidents.
\end{abstract}

Keywords: vapor cloud explosion; domino effect; cascading effect; Petri-net; escalation probability; propagation pattern

\section{Introduction}

In the petrochemical industry, it is often necessary to process or store flammable gases or liquids with high volatility. If an accident occurs at one unit containing such materials, it may have an impact on other units nearby to induce a domino effect, which refers to a series of accidents triggered by an initial event/accident. Domino effects will cause more significant human and asset losses than a primary accident (Reniers and Cozzani, 2013). Domino effects or chains of accidents in which an accident in a unit propagates into nearby units have been recognized as a priority issue among the risk and safety management, e.g., the requirements of the EU Seveso-III Directive (Directive 2012/18/EU). In process plants, there may be a large number of major hazardous installations (MHIs) containing large inventories of flammable and explosive substances, a primary accident caused by these substances is likely to escalate to a domino effect, and the safety measures like safety distances between the installations cannot completely avoid the domino effects due to restrictions such as limited

${ }^{*}$ Corresponding author. 
available land.

Among all accidents, vapor cloud explosion (VCE) is destructive. Predicting damage from vapor cloud explosions has been studied in previous works (Pritchard, 1989; Alonso et al., 2008; Salzano \& Basco, 2015). A VCE accident usually has a large extent of impact, and it is easy to trigger the domino effect. Abdolhamidzadeh et al. (2010) studied the past domino accidents and found that explosions are the most frequent cause of domino effect (57\%), and among the explosions VCE (vapor cloud explosion) has been the most frequent cause (84\%). In literature, there are some studies about the domino effect of VCE. For example, Cozzani and Salzano (2004a, 2004b) revised data on process equipment damage caused by blast waves, and derived specific probit models for several categories of process equipment. Cozzani and Salzano (2004c) proposed thresholds values of overpressure for the damage to equipment caused by blast waves. Salzano and Cozzani (2005) revised available quantitative assessment models of damage probability to plant equipment caused by pressure waves generated by a vapor cloud explosion. Zhang \& Jiang (2008) revised more reliable data to build a quantitative relationship between damage probability and damage degrees of equipment caused by overpressure, and develop reliable probit models. Antonioni et al. (2009) applied a methodology developed for the quantitative assessment of risk due to domino effect to the analysis of an extended industrial area, equipment damage probability models including the probit models of VCE were applied for the identification of the final scenarios and for escalation probability assessment. Kadri et al. (2013) developed a methodology, which is based on the probabilistic models and the physical equations, for quantitative assessment of domino effects caused by fire and explosion in industrial sites. Mukhim et al (2017) presented a probit method to estimate the probability of accident escalation in chemical process industries due to domino effect triggered by overpressure, based on a larger data set, a finer level of classification of the equipment and a different method of linking the qualitative description of the damage to the quantitative probability.

A VCE accident may result in damage to nearby units and lead to secondary VCE. The overpressures generated by secondary VCE accidents may in turn trigger other VCEs. This propagation of VCEs is called the cascading effect of VCE, which is capable of causing severe damages to human, assets, and the environment. This cascading effect of VCE accidents has happened in reality. For example, on 23 October 1989, a vapor cloud explosion occurred at the Phillips 66 Company polyethylene production plant near Pasadena, Texas (U.S. Fire Administration, 2011). The accident resulted in 23 deaths, 1 missing and more than 130 injured. The vapor cloud resulted as a sudden gas release of an estimated 85,000 lbs of a flammable gas mixture through an 8 -inch open valve. The vapor cloud plus additional flammable gas inside tanks at the valve source exploded with a force equivalent to 2.4 tons of TNT based on blast damage. Two isobutene storage tanks exploded 10 to 15 minutes later. A third explosion occurred 15 to 30 minutes later when a polyethylene plant reactor failed catastrophically. The two polyethylene production plants covering 16 acres near the source of the blast were destroyed. On 27 June 1997, a catastrophic accident happened in Beijing Dongfang Chemical plant of China (China Academy of Safety Science, 2005). A great deal of naphtha spilled over from a tank roof as an operator opened the wrong valve. The explosive gas mixed with volatilized naphtha and air was ignited to form a severe vapor cloud explosion, which resulted in successive explosions of 
nearby ethylene tank A and ethylene tank B (thousands of cubic meters of ethylene are stored in each tank). This accident killed 9 people and injured 39 others, 20 tanks were destroyed.

In the past studies, there are few attempts made to model and assess the cascading effect of VCE, especially the total probability of each unit with mutual influences under the domino effect, although several researchers have studied the propagation of accidents, for example, Khakzad et al. (2013) provided a Bayesian network based approach to model domino effect propagation patterns and to estimate the domino effect probability and Yuan et al. (2016) developed a methodology for the probability estimation of a series of dust explosions based on Bayesian network.

The present study aims to develop a novel methodology using Petri-net (PN) for modeling the propagation of VCEs and estimating the probabilities of VCE accidents. Petri-net was originally proposed in Carl Adam Petri's dissertation (Petri, 1966), and from then on it is widely used to model and analyze various systems such as communication, manufacturing, and transportation systems. Petri-nets are a graphical and mathematical modeling tool composed of places, transitions, and arcs. They are a promising tool for describing and studying relationships between parts of a systems which are characterized as being concurrent, asynchronous, distributed, parallel, nondeterministic, and/or stochastic (Murata, 1989). In addition to modeling of systems, tokens are used in Petri-nets to simulate the dynamic and concurrent activities of a system. Petri-net is utilized to model the impacts of one unit on another under VCE accidents in this study. To model and analyze the states and their changes of VCE propagation among many units, an improved Petri-net is proposed based on the basic Petri-net.

This paper is organized as follows: Section 2 introduces the domino effect of VCE, and problems in probability analysis of VCE cascading effect. In Section 3, the definition and the improving of Petri-net as well as the modeling and analysis process of VCE cascading effect are provided. In Section 4, an example illustrates the proposed approach. Finally, the conclusions drawn from this work are presented in Section 5.

\section{Cascading effect of VCE}

\subsection{Models of domino effect of VCE}

If an explosive mixture of flammable gas and air is ignited, it is likely to form a VCE accident. If an equipment nearby is in the impact extent of VCE overpressure, it may fail and a secondary accident may occur. To determine whether a nearby unit may fail, the overpressures exerted by the primary VCE event on the nearby units are compared with predefined threshold values. The overpressures above the relevant thresholds are strong enough to cause credible damage to the nearby units, resulting in secondary accidents, e.g. other VCE accidents. To simplify the problem, wind direction and wind speed are not considered in this study, the gas cloud is considered around the unit after a unit is damaged.

In the literature, several researchers proposed probit models to assess the damage probability of process equipment as a consequence of a blast wave (Eisenberg et al., 1975; Khan and Abbasi, 1998; Cozzani and Salzano, 2004a; Zhang and Jiang, 2008; Mukhim et al., 2017). Generally, the probit value $\operatorname{Pr}$ can be obtained using Eq. (1):

$$
\operatorname{Pr}=a+b \ln (\Delta P)
$$

Where, $a$ and $b$ are probit coefficients determined using experimental data and regression methods, 
and $\Delta P$ is overpressure $(\mathrm{Pa})$ in the case of explosion.

After $\mathrm{Y}$ is determined, the escalation probability, $P_{e s c}$, could be calculated as Eq. (2):

$$
P_{\text {esc }}=\phi(P r-5)
$$

Where, $\phi$ is the cumulative density function of standard normal distribution.

In this study, the probit methods proposed by Cozzani and Salzano (2004a) are used to obtain the probit value for overpressure. For atmospheric equipment, $\operatorname{Pr}$ can be determined according to Eq. (3).

$$
\operatorname{Pr}=-18.96+2.44 \ln (\Delta P)
$$

And for pressurized equipment, $\operatorname{Pr}$ can be determined according to Eq. (4).

$$
\operatorname{Pr}=-42.44+4.33 \ln (\Delta P)
$$

VCE accidents may propagate from one unit to another. The VCE accidents of secondary units in the domino effect not only intensify the accident, but also help the domino effect escalate to tertiary units. The overpressures originating from secondary VCE accidents in turn trigger other accidents. The propagation of VCEs may form a cascading effect.

\subsection{Deficiency in probability analysis of domino effect}

During the propagation of VCE accidents, one unit may be in the impact extent of the VCE accident of another unit, and the VCE accident of this unit may also impact that unit, that is, correlation exists in probability calculation of domino effect. These characteristics of VCE propagation make the probit methods cannot be directly used for probability analysis of VCE cascading. In previous studies (e.g. Khakzad et al., 2013), only most possible propagation patterns are considered during the analysis of domino effect. However, for total probability analysis, any possible propagation should be considered. In the tank farm shown in Fig. 1 (a), if a VCE accident occurs at TK1, TK2 is most likely to be damaged under the overpressure from the VCE accident of TK1 according to the overpressure between the tanks and the propagation paths. But to determine the probability of VCE at TK2, the probabilities of other propagation paths which are shown in Fig. 1(b), including TK1 $\rightarrow \mathrm{TK} 3 \rightarrow \mathrm{TK} 2$, $\mathrm{TK} 1 \rightarrow \mathrm{TK} 4 \rightarrow \mathrm{TK} 2$, and $\mathrm{TK} 1 \rightarrow \mathrm{TK} 3 \rightarrow \mathrm{TK} 4 \rightarrow \mathrm{TK} 2$, should be calculated, although the probabilities of some propagation paths are very small. It should be noted that the VCE probability of TK2 should be also taken into account in calculating the probabilities of VCE accidents at TK3 or TK4.
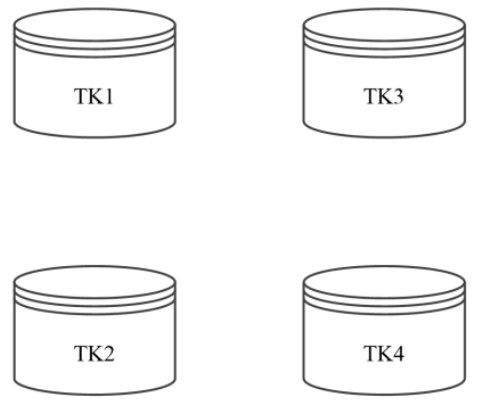

(a)

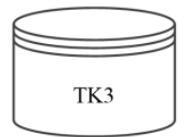

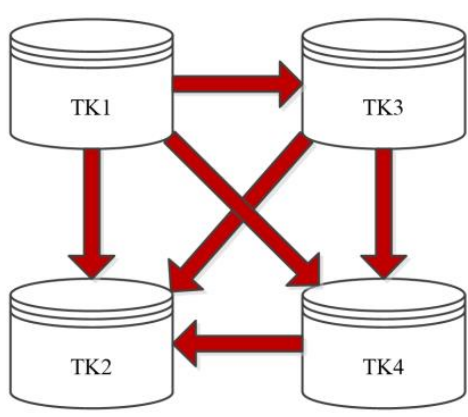

(b)

Fig. 1 Layout of four tanks (a) and possible propagations from TK1 to TK2 (b)

If there are many units that may influence each other under the overpressure of VCE accidents in an area, the total probability analysis of domino effect will be very complex. 
The main aim of this work is to introduce a new methodology based on Petri-net to analyze this cascading effect of VCE accidents. Although there are mutual influences between the units on probability calculation, there is no interaction between them during a propagation process. Thus, the probabilities can be obtained through simulation of propagation processes.

\section{Petri-net based analysis approach}

\subsection{Definitions and rules of Petri-net}

(i) Definition of Petri-net

Petri-nets are mathematical modeling tools used to analyze and simulate concurrent systems (Murata, 1989). The system is modeled as a directed graph with two sets of nodes: the set of places representing state or system objects and the set of events or transitions determining the dynamics of the system.

A Petri net (PN) is a 5-tuple:

$$
P N=(P, T, I, O, M)
$$

Where:

$P=\left\{p_{1}, p_{2}, \ldots, p_{n}\right\}$ is a finite set of places.

$T=\left\{t_{1}, t_{2}, \ldots, t_{m}\right\}$ is a finite set of transitions.

$I$ : an input function, $(P \times T) \rightarrow N$, where $N$ is the set of non-negative integer numbers. The value $I(p$, $t$ ) is the number of (directed) arcs from the place $p$ to the transition $t$.

$O$ : an output function, $(T \times P) \rightarrow N$, where the value $O(t, p)$ is the number of arcs from the transition $t$ to the place $p$.

$M: P \rightarrow N$ is the marking of a Petri-net. A marking $M$ of a Petri net with $n$ places is a $(1 \times n)$ vector and associates with each place a certain number of tokens which are usually represented by means of dots inside the circles. The initial marking $M_{0}$ represents the initial state of the system. The marking at place $p_{i}$ can also be denoted as $M\left(p_{i}\right)$.

Usually, places are represented with circles, and transitions are represented with rectangles on PN graphs. The arcs (input and output functions) are represented with directed lines, and the tokens in a place are denoted as dots or a number.

A Petri net is executable. The execution of a Petri-net depends on the number and distribution of tokens in the Petri-net (Peterson, 1981). Let ${ }^{*}\left({ }^{*} p\right)$ and $t^{*}\left(p^{*}\right)$ denote the set of input places of transition $t$ (the set of input transitions of place $p$ ) and the set of output places of transition $t$ (the set of output transitions of place $p$ ), respectively. The following two rules control the execution of a Petri-net:

(a) Enabling rule: A transition is enabled if each of its input places has at least as many tokens in it as arcs from the place to the transition.

(b) Execution/firing rule: If a transition is enabled, it can fire/execute. Execution of an enabled transition $t$ at marking $M$ changes the marking into $M^{\prime}$.

$$
\begin{gathered}
M^{\prime}\left(p_{i}\right)=M\left(p_{i}\right)+x \text { for } p_{i} \in t^{\bullet} \\
M^{\prime}\left(p_{j}\right)=M\left(p_{j}\right)-y \text { for } p_{j} \in{ }^{\cdot} t
\end{gathered}
$$

Where, $x$ is the number of arcs from transition $t$ to the place $p_{i}$, and $y$ is the number of arcs from the 
place $p_{j}$ to the transition $t$.

\section{(ii) Improving of Petri-net}

Petri-net can be used for the state transition system modeling. Usually the states of a system can be represented by the places and the changing of the states can be represented by the transitions of a Petri-net. For the VCE cascading analysis, there are some special requirements for modeling the states and their changing, as the units containing flammable gas may interact each other during the cascading of VCE accidents.

If the state of VCE accident of a unit is represented by a place, and the impact of overpressure from one unit to another is represented by a transition, at most the place can only contain one token to indicate the unit is in the corresponding state, because the state (e.g. VCE accident) of this unit can only occur once. In addition, after the VCE accident of a unit has occurred, this unit will not be influenced by the VCE accidents from other units. Thirdly, the impact of one unit on any other unit must meet certain conditions, e.g. the overpressure discussed in Section 2. To model this type of state-constrained system, the Petri-net is improved in the following areas:

- Input function. $I:(P \times T) \rightarrow\{0,1\}$, there is at most one arc from a place $p$ to a transition $t$.

- Output function. $O:(T \times P) \rightarrow\{0,1\}$, there is at most one arc from a transition $t$ to a place $p$.

- Marking. $M: P \rightarrow\{0,1\}$ is the marking of a Petri-net. A place $p$ can have zero or one token.

- Enabling function $f_{e n}$ of a transition $t$. This function is introduced to represent the fact that a transition is enabled and can be executed, it needs to meet certain conditions in addition to the tokens in its input places.

- Enabling rule: A transition $t$ of a Petri-net is enabled in a marking $M$ if

$$
\begin{aligned}
& M\left(p_{i}\right)>0 \quad \text { for } p_{i} \in{ }^{\cdot} \text {, and } \\
& f_{\text {en }}(t)=\text { true, and } \\
& M\left(p_{j}\right)=0 \quad \text { for } p_{j} \in t^{*}
\end{aligned}
$$

- Execution/firing rule: After transition $t$ fires, the marking of the Petri-net changes to $M^{\prime}$, where $M^{\prime}$ is given by

$$
\begin{array}{ll}
M^{\prime}\left(p_{i}\right)=M\left(p_{i}\right) & \text { for } p_{i} \in{ }^{*} t \\
M^{\prime}\left(p_{j}\right)=1 & \text { for } p_{j} \in t^{*}
\end{array}
$$

The execution rule of a transition $t$ can be represented as Fig. 2. After the execution, unlike ordinary Petri-net that removes tokens from its input places, the improved Petri-net does not remove the tokens in its input places, as the tokens indicate the states of occurring of events.
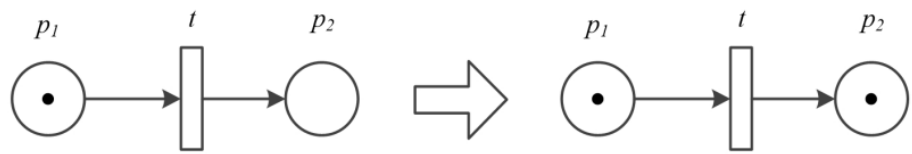

Fig. 2 Execution rule of transition $t$

\subsection{Modeling and analysis of VCE cascading effect}

Based on the improvement of Petri-net, the domino effect and the cascading effect of VCE 
accidents can be modeled. Take two units as an example. A VCE accident occurs at Unit1, the overpressure causes the failure of Unit2, and therefore results in a VCE accident at Unit2. This process is expressed in Fig. 3 (a), and is modeled by Petri-net as Fig. 3 (b). Place p1 indicates the VCE accident at Unit1, p2 represents the failure of Unit2, and p3 indicates the VCE accident at Unit2. A token in p1 indicates that a VCE accident has occurred at Unit1. Transition $\mathrm{t} 1$ indicates the impact of VCE accident at Unit1 on Unit2, and 2 represents the damage of Unit2 leads to VCE accident at Unit2. As this study focuses on VCE accidents, the Petri-net model can be simplified as shown in Fig. 3 (c), where p2 indicates the VCE accident at Unit2, and $\mathrm{t} 1$ which combines $\mathrm{t} 1$ and $\mathrm{t} 2$ in Fig. 3 (b) represents that overpressure from Unit1 leads to VCE accident at Unit2.

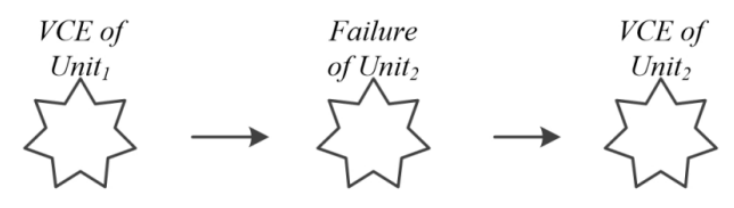

(a)

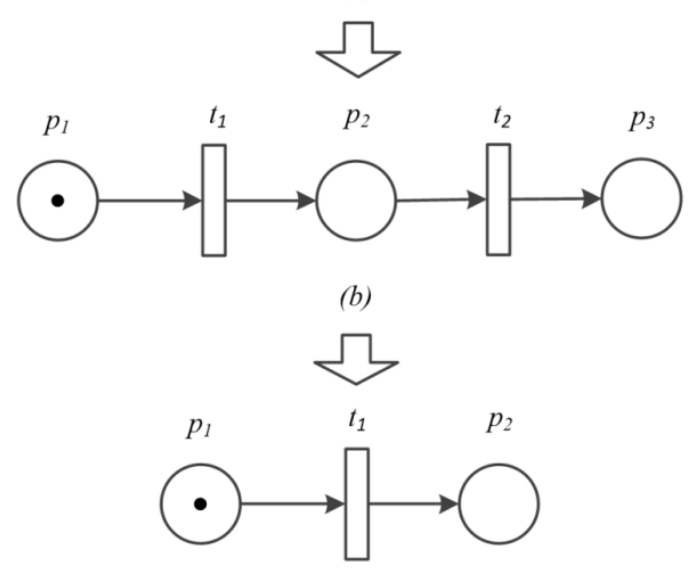

(c)

Fig. 3 Modeling of VCE domino effect based on Petri-net

Based on the modeling of VCE domino effect, the probabilities of the cascading effect of VCE accidents can be analyzed through simulation. The flowchart of the analysis process is shown in Fig. 4.

Step 1: Establish the Petri-net model for VCE cascading effect analysis. Estimate the overpressure of VCE accident between any two units, and model the impact of overpressure by Petri-net if it exceeds the threshold of VCE domino effect.

Step 2: Initialize variables of the simulation analysis of VCE cascading effect, e.g. set the number of simulation analysis SimCnt, let the trial index $i d x=0$, and set the number of VCE accident at any unit to zero, etc..

Step 3: Perform a trial of VCE cascading. This includes the following sub-steps:

Step 3.1: Initialize the trial states. Add the value of the trial index $i d x$ by one. Clear tokens of all places and put a token in the place indicating the primary VCE accident.

Step 3.2: Sample and determine the values of the enabling function $f_{e n}$ of the transitions. In this study, function $f_{\text {en }}$ is determined as follows, 


$$
\begin{aligned}
f_{e n} & =f_{1} \text { and } f_{2} \\
f_{1}= & \text { true if normalnum }(0,1)<P r-5, \\
& \text { false otherwise; } \\
f_{2}= & \text { true if random }()<P_{v c e}, \\
& \text { false otherwise. }
\end{aligned}
$$

where, normalnum $(x, y)$ is a function to generate a random number satisfying normal distribution with a mean value of $x$ and a variance of $y$, and $\operatorname{random}()$ is a function that randomly generates a number between 0 and 1 .

Function $f_{l}$ is utilized to meet the requirement of Eq. (2), and function $f_{2}$ is adopted to satisfy the requirement of VCE accident likelihood/probability $P_{v c e}$ after a unit is damaged.

Step 3.3: Execute the Petri-net model until no transition is enabled. Execute the model according to the enabling rule and the execution rule of the Petri-net. Tokens are put into corresponding places according to the execution to indicate the occurrence of VCE accidents. If on transition is enabled, it means that the VCE cascading process ends.

Step 3.4: Record the results of the trial. The final marking of the Petri-net model which indicates VCE accidents at different units needs to be recorded, and the number of VCE accident at any unit should be counted according to the final marking. In addition, to analyze the mutual influence between the units, the source of the VCE accident at a unit can also be recorded.

Step 4: If the number of trials is less than the given number of simulation analysis SimCnt, go back to the beginning of Step 3 (Step 3.1) to perform the next trial, otherwise, calculate the probabilities of VCE accidents at all units, and analyze the impacts between the units. 


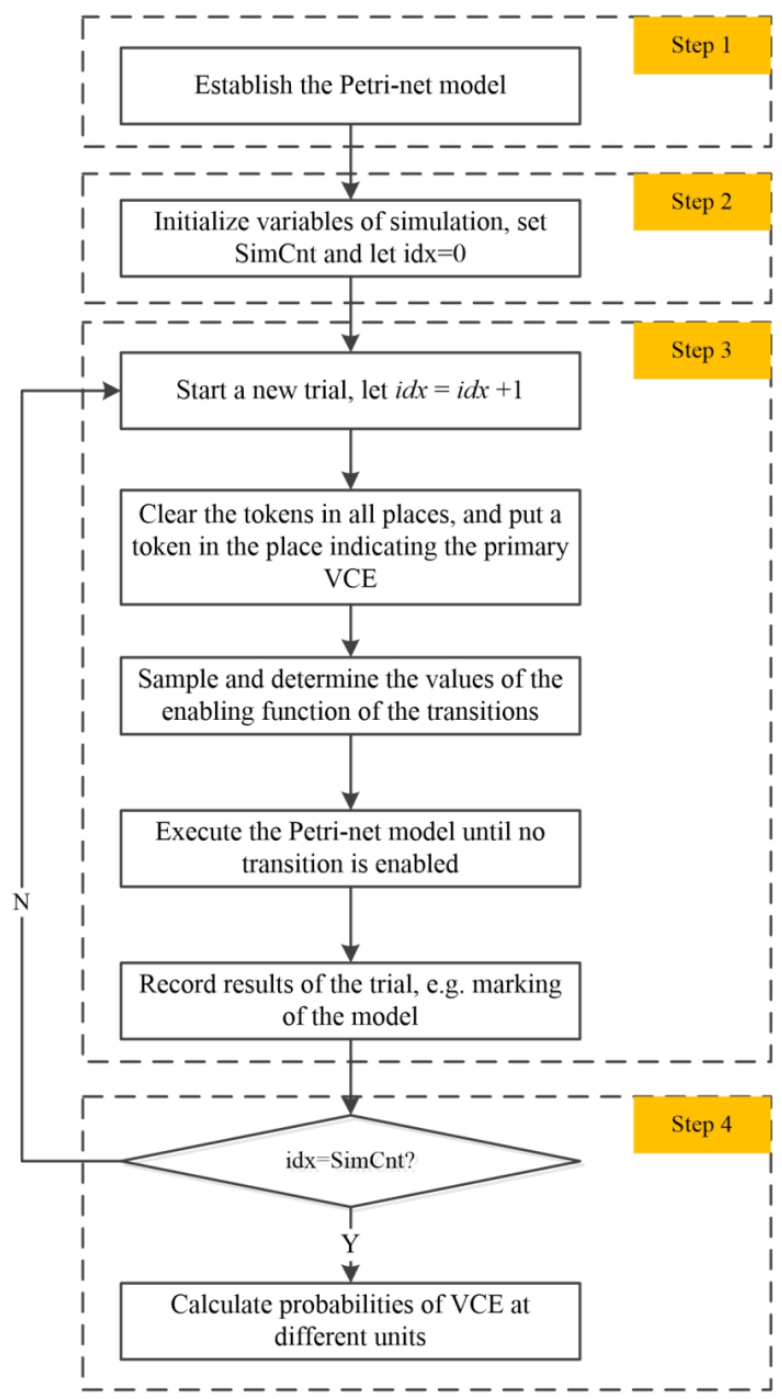

Fig. 4 Flowchart of VCE cascading effect analysis

\section{An example}

In the study of Cozzani and Salzano (2004b), domino effects in atmospheric tanks containing fuels with high volatility (gasoline) are analyzed. This case is utilized to illustrate the VCE cascading analysis approach proposed in this study.

To simplify the problem, only 6 of the 8 tanks are considered in this study. The layout of the tanks is shown in Fig. 5. 

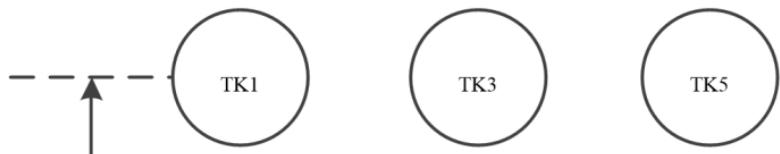

$$
50 \mathrm{~m}
$$

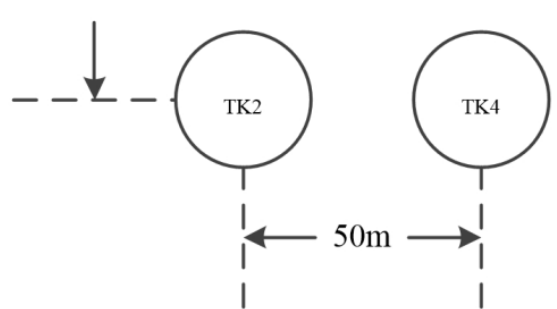

Fig. 5 Layout of the gasoline tanks

Each tank contains gasoline with the capacity of 2,000 metric tons. After a storage tank is damaged through the domino effect, it may result in a secondary accident such as pool fire and VCE. Assume that the VCE probability is 0.50 after a tank is damaged. In this study, only VCE accidents are considered, and the threshold value of overpressure is selected as $\Delta P_{t h}=7 \mathrm{kPa}$ (Khakzad et al., 2013). The overpressure escalation vectors are calculated and illustrated in Table 1 (Cozzani and Salzano, 2004b).

Table 1 Overpressure escalation vectors $(\mathrm{kPa})$ (Cozzani and Salzano,2004b)

\begin{tabular}{ccccccc}
\hline & TK1 & TK2 & TK3 & TK4 & TK5 & TK6 \\
\hline TK1 & - & 10 & 10 & 8 & 4 & 4 \\
TK2 & 10 & - & 8 & 10 & 4 & 4 \\
TK3 & 10 & 8 & - & 10 & 10 & 8 \\
TK4 & 8 & 10 & 10 & - & 8 & 10 \\
TK5 & 4 & 4 & 10 & 8 & - & 10 \\
TK6 & 4 & 4 & 8 & 10 & 10 & - \\
\hline
\end{tabular}

Suppose a VCE accident occurs at TK1, the VCE cascading effect Petri-net model which is shown in Fig. 6 is established according to overpressure escalation vectors between the tanks. The meanings of places and transitions of the model are listed in Table 2. 


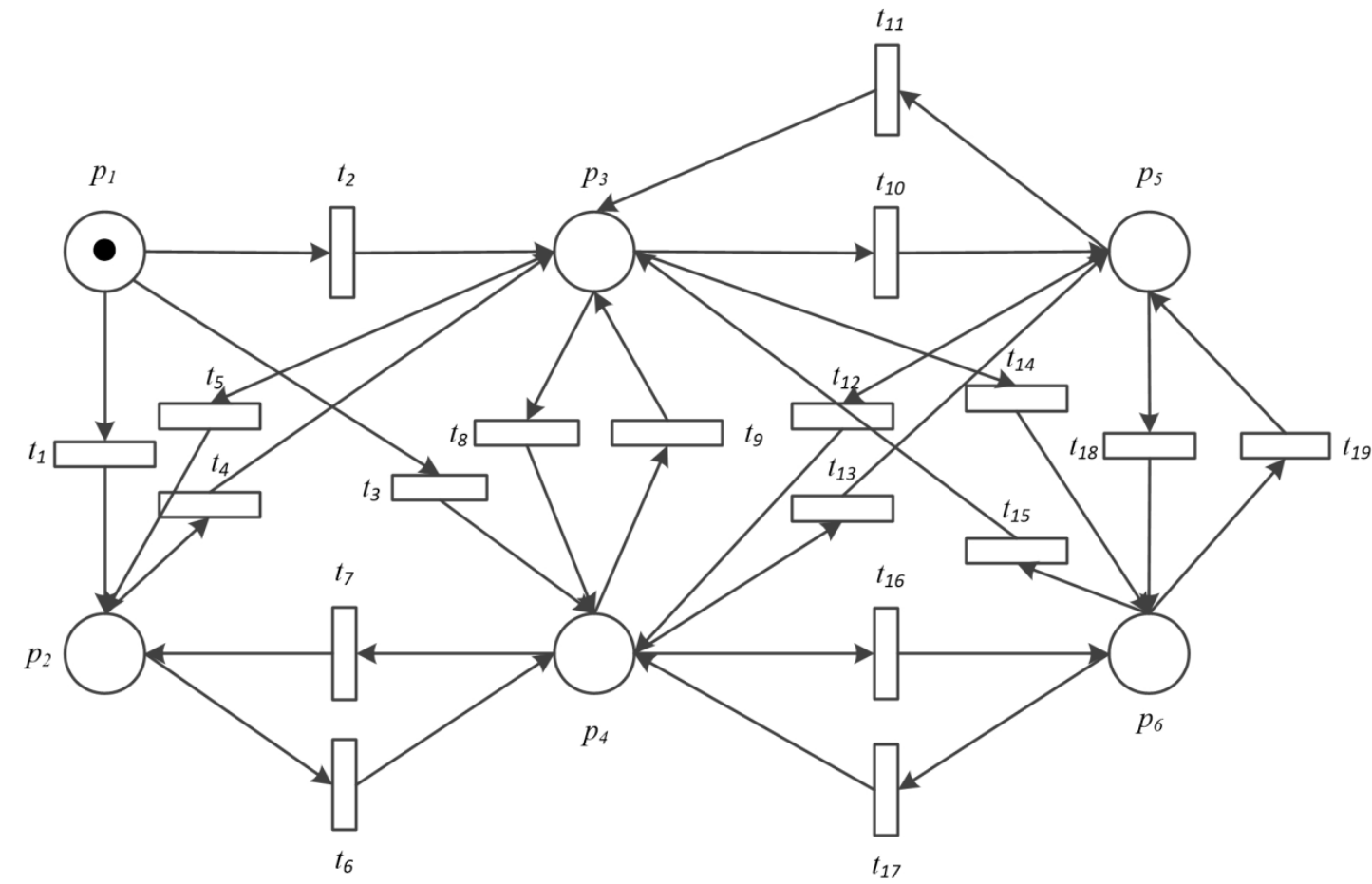

Fig. 6 Petri-net model for VCE cascading effect analysis

Table 2 Meanings of places and transitions of the Petri-net model

\begin{tabular}{llll}
\hline $\begin{array}{l}\text { Place/ } \\
\text { Transition }\end{array}$ & Meanings & $\begin{array}{l}\text { Place/ } \\
\text { Transition }\end{array}$ & Meanings \\
\hline$p_{1}$ & VCE at TK1 & $t_{8}$ & Overpressure from TK3 impacts TK4 \\
$p_{2}$ & VCE at TK2 & $t_{9}$ & Overpressure from TK4 impacts TK3 \\
$p_{3}$ & VCE at TK3 & $t_{10}$ & Overpressure from TK3 impacts TK5 \\
$p_{4}$ & VCE at TK4 & $t_{11}$ & Overpressure from TK5 impacts TK3 \\
$p_{5}$ & VCE at TK5 & $t_{12}$ & Overpressure from TK5 impacts TK4 \\
$p_{6}$ & VCE at TK6 & $t_{13}$ & Overpressure from TK4 impacts TK5 \\
$t_{1}$ & Overpressure from TK1 impacts TK2 & $t_{14}$ & Overpressure from TK3 impacts TK6 \\
$t_{2}$ & Overpressure from TK1 impacts TK3 & $t_{15}$ & Overpressure from TK6 impacts TK3 \\
$t_{3}$ & Overpressure from TK1 impacts TK4 & $t_{16}$ & Overpressure from TK4 impacts TK6 \\
$t_{4}$ & Overpressure from TK2 impacts TK3 & $t_{17}$ & Overpressure from TK6 impacts TK4 \\
$t_{5}$ & Overpressure from TK3 impacts TK2 & $t_{18}$ & Overpressure from TK5 impacts TK6 \\
$t_{6}$ & Overpressure from TK2 impacts TK4 & $t_{19}$ & Overpressure from TK6 impacts TK5 \\
$t_{7}$ & Overpressure from TK4 impacts TK2 & & \\
\hline
\end{tabular}

Let's trace the propagation of VCE accidents to illustrate the analysis approach. According to the overpressure escalation vectors shown in Table 1, and the probit method shown in Eq. (3), the probits between the tanks can be obtained as shown in Table 3.

Table 3 Probits between the tanks

\begin{tabular}{ccccccc}
\hline & TK1 & TK2 & TK3 & TK4 & TK5 & TK6 \\
\hline TK1 & - & 3.513 & 3.513 & 2.969 & - & - \\
\hline
\end{tabular}




\begin{tabular}{rcccccc}
\hline TK2 & 3.513 & - & 2.969 & 3.513 & - & - \\
TK3 & 3.513 & 2.969 & - & 3.513 & 3.513 & 2.969 \\
TK4 & 2.969 & 3.513 & 3.513 & - & 2.969 & 3.513 \\
TK5 & - & - & 3.513 & 2.969 & - & 3.513 \\
TK6 & - & - & 2.969 & 3.513 & 3.513 & - \\
\hline
\end{tabular}

According to the enabling rule of the Petri-net discussed in analysis Step 3.2, a sample of the normal distribution random numbers and the uniform distribution random numbers of the transitions are obtained and listed in Table 4.

Table 4 A sample of the random numbers for enabling the transitions

\begin{tabular}{lll}
\hline Transition & Normal distribution number & Uniform distribution number \\
\hline $\mathrm{t} 1$ & -1.827 & 0.225 \\
$\mathrm{t} 2$ & -2.420 & 0.718 \\
$\mathrm{t} 3$ & -0.063 & 0.984 \\
$\mathrm{t} 4$ & -0.088 & 0.280 \\
$\mathrm{t} 5$ & 1.239 & 0.981 \\
$\mathrm{t} 6$ & -1.869 & 0.066 \\
$\mathrm{t} 7$ & -0.364 & 0.741 \\
$\mathrm{t} 8$ & 0.111 & 0.852 \\
$\mathrm{t} 9$ & 0.004 & 0.040 \\
$\mathrm{t} 10$ & 1.162 & 0.604 \\
$\mathrm{t} 11$ & -1.507 & 0.349 \\
$\mathrm{t} 12$ & 0.362 & 0.257 \\
$\mathrm{t} 13$ & 0.759 & 0.839 \\
$\mathrm{t} 14$ & -0.762 & 0.766 \\
$\mathrm{t} 15$ & -1.890 & 0.352 \\
$\mathrm{t} 16$ & -1.580 & 0.182 \\
$\mathrm{t} 17$ & 1.216 & 0.547 \\
$\mathrm{t} 18$ & 1.903 & 0.348 \\
$\mathrm{t} 19$ & 0.198 & 0.231 \\
\hline & &
\end{tabular}

Put a token in place p1, and execute the Petri-net model, we can get the execution process as shown in Table 5:

Table 5 Execution process of the Petri-net model

\begin{tabular}{lll}
\hline No. & Marking & Executed transition \\
\hline 0 & $(1,0,0,0,0,0)$ & \\
1 & $(1,1,0,0,0,0)$ & $\mathrm{t} 1$ \\
2 & $(1,1,0,1,0,0)$ & $\mathrm{t} 6$ \\
3 & $(1,1,0,1,0,1)$ & $\mathrm{t} 16$ \\
\hline
\end{tabular}


From the execution process we can obtain the propagation pattern of the VCE accidents that the VCE accident at TK1 propagates to TK2, then to TK4, and finally to TK6. It should be noticed that the VCE accident at TK4 is caused by the VCE at TK2 as the executed transition is t6. The tank TK3 is damaged but there is no VCE at TK3.

Similarly, other propagation patterns of the VCE accidents can also be obtained. For example, if the sample of the normal distribution random number and the uniform distribution random number of the transitions are obtained as listed in Table 6, we can obtain the process shown in Table 7.

Table 6 Another sample of the random numbers for enabling the transitions

\begin{tabular}{lll}
\hline Transition & Normal distribution number & Uniform distribution number \\
\hline $\mathrm{t} 1$ & 0.622 & 0.526 \\
$\mathrm{t} 2$ & -0.560 & 0.323 \\
$\mathrm{t} 3$ & -2.545 & 0.090 \\
$\mathrm{t} 4$ & -0.229 & 0.279 \\
$\mathrm{t} 5$ & 0.267 & 0.448 \\
$\mathrm{t} 6$ & 1.854 & 0.079 \\
$\mathrm{t} 7$ & -1.549 & 0.361 \\
$\mathrm{t} 8$ & -0.167 & 0.415 \\
$\mathrm{t} 9$ & -1.366 & 0.522 \\
$\mathrm{t} 10$ & 0.341 & 0.334 \\
$\mathrm{t} 11$ & 2.363 & 0.531 \\
$\mathrm{t} 12$ & 0.704 & 0.016 \\
$\mathrm{t} 13$ & -0.153 & 0.257 \\
$\mathrm{t} 14$ & -1.899 & 0.568 \\
$\mathrm{t} 15$ & -1.014 & 0.975 \\
$\mathrm{t} 16$ & 0.672 & 0.150 \\
$\mathrm{t} 17$ & 2.508 & 0.606 \\
$\mathrm{t} 18$ & 0.824 & 0.812 \\
$\mathrm{t} 19$ & 1.680 & 0.371 \\
\hline
\end{tabular}

Table 7 Another execution process of the Petri-net model

\begin{tabular}{lll}
\hline No. & Marking & Executed transition \\
\hline 0 & $(1,0,0,0,0,0)$ & \\
1 & $(1,0,0,1,0,0)$ & t3 \\
2 & $(1,1,0,1,0,0)$ & t7 \\
\hline
\end{tabular}

In this condition, the VCE accidents propagate from TK1 to TK4, and then to TK2. There is no VCE accident occurring at other tanks.

After $10^{5}$ trials, the VCE number of times and the VCE probabilities of all tanks are determined and shown in Table 8. Under the circumstance that a VCE accident occurs at TK1, the probability of 
VCE accident at TK2 is 0.03497 , with 0.03430 resulting from TK1, $2.6 \times 10^{-4}$ caused by TK3, and $4.1 \times 10^{-4}$ from TK4. Similarly, the probability of VCE accident at any tank and the source of the accident are obtained.

Table 8 VCE probability of all tanks given a primary VCE at TK1

\begin{tabular}{lllllllll}
\hline & Probability & Times & \multicolumn{7}{c}{ Source (Times in $10^{5}$ trials) } \\
\cline { 4 - 8 } & & & TK1 & TK2 & TK3 & TK4 & TK5 & TK6 \\
\hline TK2 & 0.03497 & 3497 & 3430 & - & 26 & 41 & 0 & 0 \\
TK3 & 0.03531 & 3531 & 3439 & 44 & - & 47 & 0 & 1 \\
TK4 & 0.0129 & 1290 & 1086 & 107 & 94 & - & 1 & 2 \\
TK5 & 0.00145 & 145 & 0 & 0 & 128 & 16 & - & 1 \\
TK6 & 0.00109 & 109 & 0 & 0 & 41 & 59 & 9 & - \\
\hline
\end{tabular}

\section{Conclusions}

Vapor cloud explosion in the petrochemical industry is highly destructive, and has an impact on the surrounding equipment in a large extent, so that it is easy to trigger the domino effect. If an area has many units containing flammable gases or liquids with high volatility, one VCE accident may escalate to a secondary VCE accident, and the secondary VCE accident may also lead to a tertiary VCE accident, and so on. This is a cascading effect of VCE accidents, which has not been paid enough attention in the past studies.

During the propagation of VCE accidents, the units may have impacts on each other. This brings some difficulties to probabilistic analysis of the VCE accidents. In this study, a Petri-net based approach is proposed to analyze the cascading effect of VCE accidents, as Petri-net has advantages on modeling and analysis of various relationships among parts of a system. According to the requirements of VCE cascading effect analysis, the Petri-net is improved in the fields including marking, enabling rule, execution rule, and so on, to model the event states and their changes. The process of VCE cascading effect analysis is provided.

An example about VCE accidents among four tanks is utilized to illustrate the proposed VCE cascading effect analysis approach. The cascading processes representing different propagation patterns of VCE accidents are discussed and probabilities of VCE accidents at all tanks are obtained based on simulation analysis.

\section{Acknowledgments}

This work is supported by National Natural Science Foundation of China (No. 71673060).

\section{References}

Abdolhamidzadeh B., Abbasi T., Rashtchian D., Abbasi S. A., (2010). Domino effect in process-industry-an inventory of past events and identification of some patterns. Journal of Loss Prevention in Process Industries 24 (5), 575-593.

Alonso F. D., Ferradas E. G., Sanchez T. de Jesus J., et al. (2008). Consequence analysis to determine the damage to humans from vapour cloud explosions using characteristic curves. Journal of 
Hazardous Materials 150, 146-152.

Antonioni G., Spadoni G., Cozzani V., (2009). Application of domino effect quantitative risk assessment to an extended industrial area. Journal of Loss Prevention in the Process Industries 22, 614-624.

China Academy of Safety Science. (2005). Hazardous chemical accident cases (in Chinese). Chemical Industry Press, Beijing, China.

Cozzani V., Gubinelli G., Salzano E., (2006). Escalation thresholds in the assessment of domino accidental events. Journal of Hazardous Materials A129, 1-21.

Cozzani V., Salzano E., (2004a). The quantitative assessment of domino effects caused by overpressure Part I. Probit models. Journal of Hazardous Materials A107, 67-80.

Cozzani V., Salzano E., (2004b). The quantitative assessment of domino effects caused by overpressure Part II. Case studies. Journal of Hazardous Materials A107, 81-94.

Cozzani V., Salzano E., (2004c). Threshold values for domino effects caused by blast wave interaction with process equipment. Journal of Loss Prevention in the Process Industries 17, 437-447.

Eisenberg N. A., Lynch C. J., Breeding R. J., (1975). Vulnerability model: a simulation system for assessing damage resulting from marine spills. In: Rep. CG-D-136-75, Enviro Control Inc Rockville MD.

Kadri F., Châtelet E., Chen G., (2013). Method for quantitative assessment of the domino effect in industrial sites. Process Safety and Environmental Protection 91, 452-462.

Khakzad N., Khan F., Amyotte P., Cozzani V., (2013). Domino Effect Analysis Using Bayesian Networks. Risk Analysis 33(2), 292-306.

Khan F. I., Abbasi S. A., (1998). Models for domino analysis in chemical process industries, Process Safety Progress 17, 107-123.

Mukhim E. D., Abbasi T., Tauseef S. M., Abbasi S. A., (2017). Domino effect in chemical process industries triggered by overpressure-Formulation of equipment-specific probits. Process Safety and Environmental Protection 106, 263-273.

Murata T., (1989). Petri nets: Properties, analysis and applications. Proceedings of the IEEE 77(4), 541-580.

Peterson J. L., (1981). Petri net theory and the modeling of systems. Prentice-Hall, Inc., Englewood Cliffs, N. J., USA.

Petri C. A., (1966). Communication with Automata. New York: Griffiss Air Force Base. Technical Report No. RADC- TR-65-377, Volume 1, Supplement 1.

Pritchard D. K., (1989). A review of methods for predicting blast damage from vapour cloud explosions. Journal of Loss Prevention in Process Industries 2, 187-193.

Reniers G., Cozzani V., (2013). Domino Effects in the Process Industries: Modeling, Prevention and Managing. Elsevier, Amsterdam, The Netherlands.

Salzano E., Basco A., (2015). Simplified model for the evaluation of the effects of explosions on industrial target. Journal of Loss Prevention in the Process Industries 37, 119-123.

Salzano E., Cozzani V., (2005). The analysis of domino accidents triggered by vapor cloud explosions. Reliability Engineering and System Safety 90, 271-284. 
U.S. Fire Administration. (2011). Phillips Petroleum Chemical Plant Explosion and Fire. Technical Report Series USFA-TR-035. Available at: http://www.usfa.dhs.gov/.

Yuan Z., Khakzad N., Khan F., Amyotte P., (2016). Domino effect analysis of dust explosions using Bayesian networks. Process Safety and Environmental Protection 100, 108-116.

Zhang M., Jiang J., (2008). An improved probit method for assessment of domino effect to chemical process equipment caused by overpressure. Journal of Hazardous Materials 158, 280-286. 